\title{
Optimizer Smart in the treatment of moderate-to-severe chronic heart failure
}

\author{
Courtney M Campbell*,1, Rami Kahwash ${ }^{1}$ \& William T Abraham ${ }^{1}$ \\ ${ }^{1}$ Department of Internal Medicine, Division of Cardiovascular Medicine, The Ohio State University Wexner Medical Center, 200 \\ Davis Heart \& Lung Institute, 473 W 12th Ave, Columbus, OH 43210, USA \\ *Author for correspondence: courtney.campbell@osumc.edu
}

Cardiac contractility modulation, also referred to as $\mathrm{CCM}^{\top \mathrm{TM}}$, by the Optimizer Smart device is an innovative intracardiac device-based therapy that has been recently US FDA-approved for the treatment of patients with chronic heart failure, left ventricular ejection fraction (LVEF) between 25 and 45\%, QRS $<130$ ms who remain symptomatic despite optimal medical therapy. Clinical trials demonstrate that CCM therapy is safe and effective in reducing heart failure hospitalization and improving heart failure symptoms, quality of life and functional performance. This novel device-based therapeutic offers benefits to patients who do not otherwise qualify for cardiac resynchronization therapy. CCM expands the indication beyond the traditional LVEF cutoff of $35 \%$ to a newer group including patients who fall in midrange LVEF group, up to $45 \%$.

\section{Graphical abstract:}
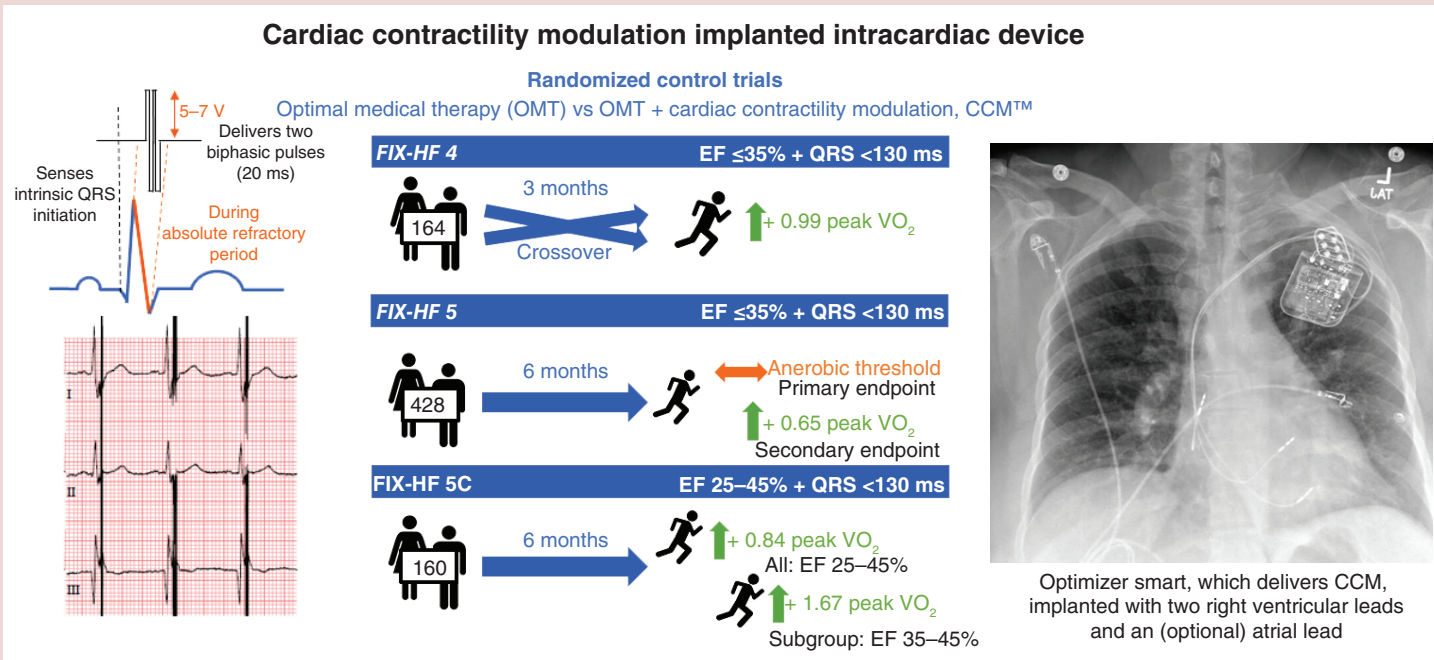

Campbell CM, Kahwash R, Abraham WT. Optimizer Smart in the treatment of moderate-to-severe chronic heart failure. Future Cardiology 16(1), 13-25 (2020).

First draft submitted: 1 August 2019; Accepted for publication: 12 November 2019; Published online: 11 December 2019

Keywords: cardiac contractility modulation $\bullet$ heart failure $\bullet$ intracardiac device $\bullet$ optimizer

Heart failure affects over 6 million adults in the USA with projections for increases to over 8 million by 2020 [1]. Approximately, $50 \%$ of people diagnosed with heart failure die within 5 years [1]. Our current medical management is inadequate to address this growing public health crisis. The medication regimen for systolic heart failure is strikingly limited: consisting of $\beta$-blockers, angiotensin-converting enzyme inhibitors or angiotensin receptor blockade and mineralocorticoid receptor antagonists. Diuretics only provide symptomatic relief. Sacubitril/valsartan and ivabradine are the only new medications for heart failure in two decades. 
Cardiac resynchronization therapy (CRT) offers a device-based therapy for patients with ejection fraction (EF) of $\geq 35 \%$ and wide QRS ( $>130 \mathrm{~ms}$ in left bundle-branch pattern) that improves cardiac function, quality of life and decreases hospitalization and morbidity [2-4]. For the majority of heart failure patients, CRT device is not indicated: only a third of heart failure patients meet criteria [5]. The implantable cardiac contractility modulation, $\mathrm{CCM}^{\mathrm{TM}}$, fills this device-based therapeutic gap. CCM devices received the European Conformity (CE) mark in October 2016 and recently received the US FDA approval in March 2019. CCM devices are included in the European Society of Cardiology 2016 Heart Failure Guidelines, and CCM inclusion in the next update of the American Heart Association and American College of Cardiology guidelines is anticipated [6,7].

CCM is an innovative intracardiac device-based therapy that has been evaluated in the treatment of patients with chronic heart failure, left ventricular ejection fraction (LVEF) $\geq 25$ and $\leq 45 \%$, QRS $<130 \mathrm{~ms}$ who remain symptomatic despite optimal medical therapy (OMT) [8-10]. In this patient population, clinical trials [8-10] showed that CCM therapy is safe and effective in reducing heart failure hospitalization and improving heart failure symptoms, quality of life and functional performance.

CCM consists of a specialized implantable pulse generator capable of delivering nonexcitatory electrical impulses to the interventricular septum through standard pacing leads [11]. These impulses are timed, so they occur during the absolute refractory periods of the myocytes' action potential. These impulses activate intracellular signals that favorably alter gene expression and enhance calcium delivery, which in turn increase myocardial contractility and cardiac efficiency without increasing metabolic demand [12].

In this review, we will discuss the development of the Optimizer Smart CE device for CCM in heart failure with an emphasis on clinical trials and future development.

\section{Cardiac contractility modulation}

Mechanism of action

The development of CCM therapy is based on observations first made in the 1960s. In the last two decades, a series of publications have expanded on these initial observations and propelled the development of the novel intracardiac CCM device. The molecular mechanism of action is not definitively understood, but many experiments have delineated how CCM changes the biology of the failing heart and the preclinical data have been previously reviewed [13]. CCM therapy increases contractility, enhances cardiac myocyte calcium handling, and improves gene expression profiles without increasing myocardial oxygen demand.

Early studies demonstrated that extracellular electrical stimulation during the absolute refractory period of calf or sheep ventricular bundles resulted in increased contractile strength and prolonged action potential duration [14]. More recent studies showed that following CCM therapy, the contraction force of healthy rabbit papillary muscles was increased within a single beat and steady state was achieved within $6-8$ beats [15]. The loss of effect within a couple of beats was similarly rapid. Similar positive inotropic effects were seen when CCM was applied to failing human left ventricular trabeculae from patients with end-stage heart failure, obtained at time of transplantation [15]. Strikingly, the average EF improved from 31 to $41 \%$ in $1 \mathrm{~h}$ and $44 \%$ after $6 \mathrm{~h}$ of CCM therapy in dogs with chronic heart failure [16]. EF improvement was maintained during 3 months of therapy [17]. In the acute setting, CCM contractility improvement was shown to be $\beta 1$-adrenergic receptor dependent [18]. In these studies, epicardial CCM stimulation was applied to isolated rabbit hearts. Basal and mid stimulation resulted in a more robust contractility response compared with apical stimulation. The magnitude of the effect correlated with increased levels of norepinephrine in samples of coronary effluent [18]. The gradient of response mirrors the distribution of sympathetic nerve fibers - the highest nerve density in basal regions and lower nerve density in apical regions [19]. Furthermore, the effects were abolished in the presence of metoprolol, a $\beta 1$-adrenergic receptor antagonist. However, CCM therapy has been effective in the setting on concomitant $\beta$-blocker therapy suggesting an additional $\beta 1$-adrenergic receptor independent mechanism [20].

CCM therapy enhances cardiac myocyte calcium handling both acutely and chronically. In early experiments using Langendorff-perfused ferret hearts, aequorin luminescence was used to show increased intracellular calcium levels after acute CCM stimulation [15]. The positive inotropic effect of CCM was blocked with the addition of ryanodine, which blocks calcium release from the sarcoplasmic reticulum $[15,20]$. Verapamil, which blocks L-type calcium channels and consequently myocyte calcium entry, also blocked CCM inotropic effects [20]. Furthermore, the inotropic effects are likely calcium-dependent as the contractility force improvement by CCM plateaued when supraphysiologic levels of calcium were used in experiments [21]. After 3 months of CCM therapy, dogs with heart failure had increased mRNA and protein levels of the SERCA-2a, phosphorylated PLN and RYR [17]. SERCA 


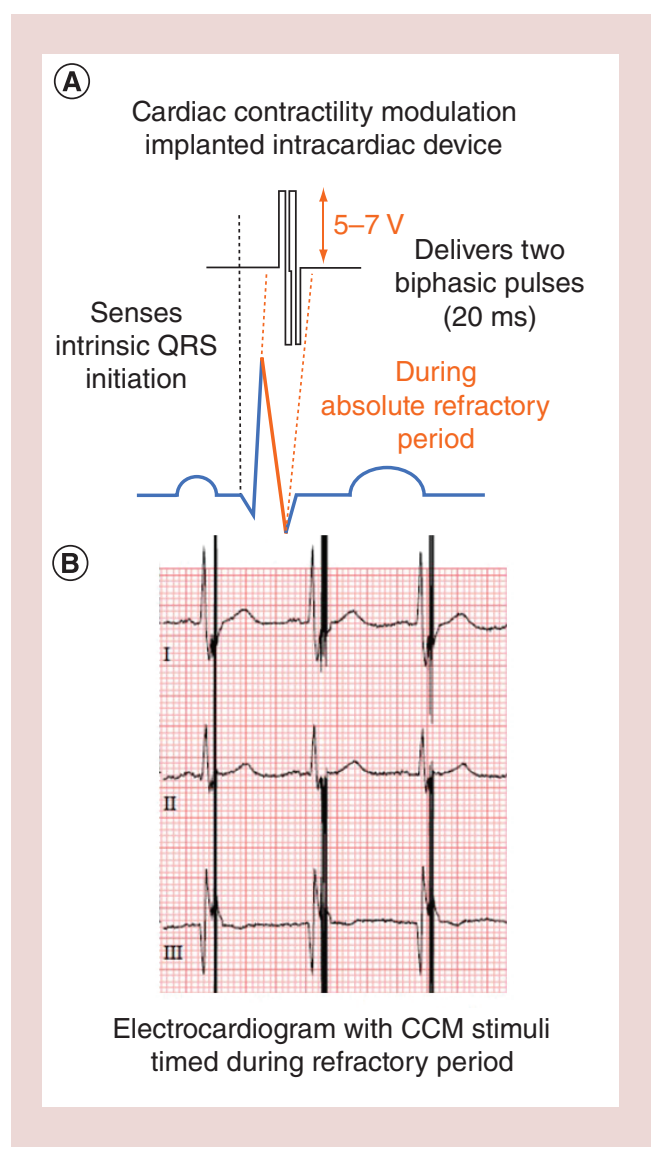

Figure 1. Cardiac contractility modulation stimulation. (A) Cardiaccontractility modulation implanted intracardiac device senses the intrinsic QRS initiation and then delivers two biphasic pulses of 5-7V over a total of $20 \mathrm{~ms}$ during the absolute refractory period. (B) Electrocardiogram leads I, II, III from a patient with an implanted CCM device demonstrating the CCM stimuli timed during the refractory period. CCM: Cardiac contractility modulation.

allows calcium to return to the sarcoplasmic reticulum and unphosphorylated PLN inhibits SERCA. Together, these protein changes augment calcium handling within the myocyte. NCX, increased in heart failure, was decreased after CCM therapy [22]. Importantly, these protein levels were comparable to healthy controls and increased compared with sham-operated dogs with failing hearts. Similar changes of mRNA expression from endomyocardial biopsies were seen in a study of 11 patients with systolic heart failure after 3 months of CCM therapy [23]. Levels of SERCA2, $P L N$ and $R Y R$ were increased. Beyond calcium-handling proteins, CCM has been shown to decrease expression of cardiac maladaptive fetal genes [17,23] and normalize expression of calcium-binding proteins [24].

Despite positive inotropic effects, CCM does not change myocardial oxygen consumption [25,26]. In an elegant study, myocardial oxygen consumption was measured before and after CCM therapy for $2 \mathrm{~h}$ in dogs with heart failure and for $30 \mathrm{~min}$ in humans [26]. Coronary blood flow was estimated using calculation based on the average peak flow velocity of the left main coronary. Oxygen content was measured from samples from the coronary artery and the coronary sinus. The product of the coronary blood flow and the arteriovenous oxygen content difference was used as a measure of myocardial oxygen consumption. In both dogs with induced heart failure and humans with chronic systolic heart failure, improvement was seen in left ventricle systolic function without an increase in myocardial oxygen consumption. In a larger study of 21 patients, ${ }^{11} \mathrm{C}$-acetate positron emission tomography imaging was used to evaluate myocardial oxygen consumption [25]. No difference was seen in oxygen consumption or work metabolic index when the CCM device was on or off. This effect is in contrast to inotropic medications, like dobutamine or milrinone, which increase contractility with an increase in myocardial oxygen consumption and result in increased mortality [27].

\section{The optimizer device}

The CCM intracardiac delivery via the Optimizer system by Impulse Dynamics (Stuttgart, Germany) is an innovative device-based therapy that has undergone improvements in each generation. Two high voltage (4.0$7.5 \mathrm{~V}$ ) biphasic electric signals of approximately $20 \mathrm{~ms}$ duration are delivered to the right ventricle septal wall during the absolute refractory period (Figure 1) [28,29]. This signal is nonexcitatory and thus does not result in a new 


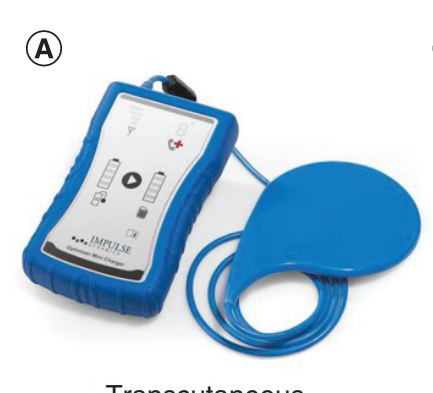

Transcutaneous home charger
(B)

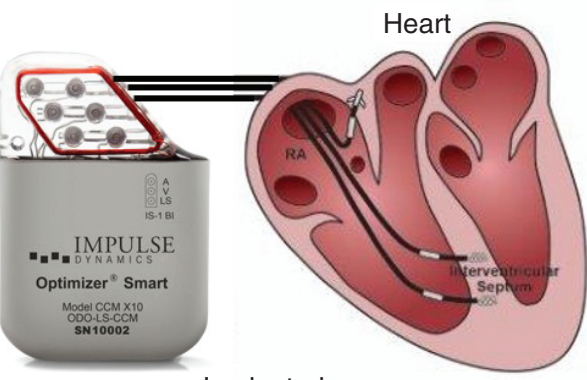

Implanted

cardiac contractility modulation

pulse generator with intracardiac leads
(C)

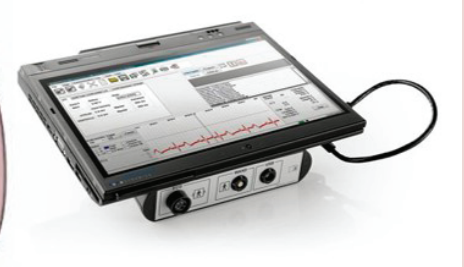

Wireless programmer

Figure 2. Optimizer system. (A) Current Optimizer System devices with transcutaneous home charger, (B) Optimizer Smart implanted cardiac contractility modulation pulse generator with intracardiac lads, and (C) wireless programmer. Images from Impulse Dynamics. Reproduced with permission from Impulse Dynamics.

contraction [30]. For comparison, this voltage is approximately 100-times the amount of energy delivered during a standard pacemaker impulse. The maximum rate of the device is programmed to $90-110$ beats per minute to avoid therapy delivery during a slow ventricular tachycardia. This therapy is delivered throughout the day for $7-12 \mathrm{~h}$ based on patient preferences and physician discretion [11].

The CCM device is similar to other intracardiac devices, such as a pacemaker or defibrillator, with a pulse generator and intracardiac leads (Figure 2) [11]. The Optimizer IV CE device consisted of three leads: atrial sensing, ventricular sensing and ventricular pacing. The most recent version of the device, Optimizer Smart CE, has only two right ventricular septal leads that are both used for sensing and pacing with an optional atrial lead [31]. Implantation of the Optimizer system is similar to other intracardiac devices [11]. In most cases, local anesthesia and conscious sedation can be used. The ventricular bipolar screw-in leads are placed transvenously in the right ventricular septum. The generator is typically placed in the right pectoral region given that many eligible patients also have another intracardiac device implanted in the left pectoral region (Figure 3). Importantly, the generator of the Optimizer system has a rechargeable battery. The patient can recharge the battery at home, which needs to be done on a weekly basis $[11,32]$. This advance is in contrast to other intracardiac devices that require periodic generator changeout procedure at the end of battery life. Compared with other intracardiac devices, the rechargeable feature is advantageous in that future procedures are not necessary. However, patients may find a weekly 40-60 min charging session to be onerous.

\section{Randomized clinical trials}

Initial human clinical studies of CCM demonstrated improved cardiac contractility with temporarily placed electrodes in patients with heart failure [29,33,34]. In early feasibility studies that were unblinded and uncontrolled, there were no overt safety issues, no proarrhythmic effects an $<\mathrm{d}$ suggested improvement in clinical heart failure parameters $[28,34]$. Here, we discuss in detail the randomized clinical trials that demonstrate CCM therapy improves exercise tolerance, as measured by peak ventilatory oxygen uptake (peak $\mathrm{VO}_{2}$ ) and by the six-minute walk test (6MWT), and quality of life, as assessed by the Minnesota Living with Heart Failure Questionnaire (MLHFQ) in patients with symptomatic moderate to severe heart failure despite OMT with normal QRS.

\section{Pilot study}

In 2006, the first prospective randomized, double-blind pilot study of CCM therapy established feasibility and safety of CCM therapy applied for 6 months [35]. Inclusion criteria included symptomatic moderate-to-severe heart failure with LVEF of $\leq 35 \%$ despite OMT and an implanted cardiac defibrillator (ICD). OMT was defined as treatment with a diuretic, a $\beta$-blocker and an angiotensin-converting enzyme inhibitor or angiotensin-receptor blocker. Exclusion criteria included CRT, peak $\mathrm{VO}_{2}<11 \mathrm{ml} / \mathrm{kg} / \mathrm{mg}$, atrial fibrillation or $\geq 8900$ premature ventricular contractions (PVCs) on a 24-h Holter monitor. Patients had to be clinical stable with no recent hospitalization within 30 days, myocardial infarction within 3 months or adjustments made to OMT within 30 days. 49 patients 


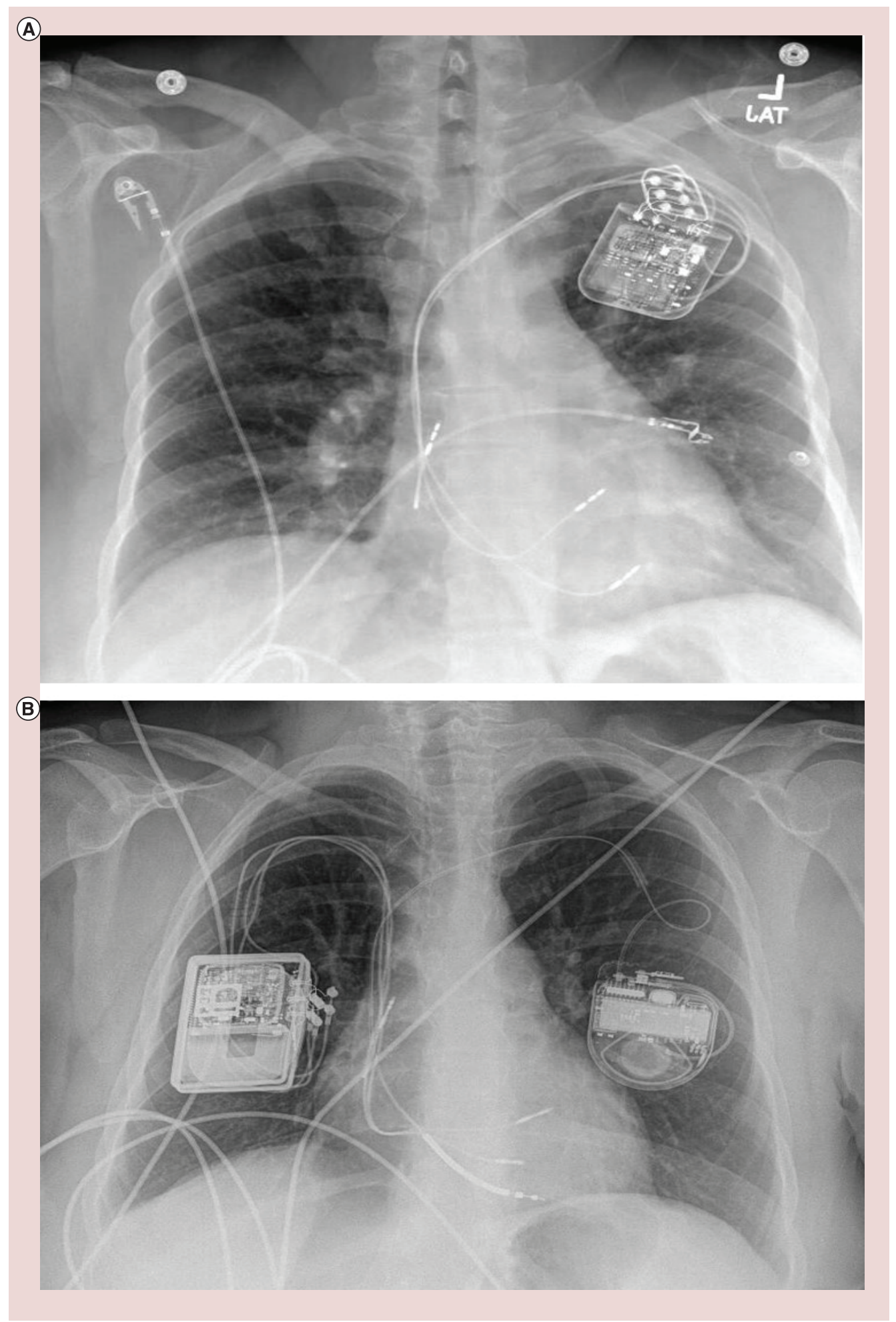

Figure 3. Implanted Optimizer Smart on chest x-ray. Chestradiographs of patient with ejection fraction $>35 \%$ implanted with Optimizer Smart cardiac contractility modulation device with pulse generator in left pectoral region and three intracardiac leads: one in the right atrium and two in the right ventricle interventricular septum (A) and patient with ejection fraction $<35 \%$ with implanted Optimize IV cardiac contractility modulation device with pulse generator in right pectoral region and three intracardiac leads: one in the right atrium and two in the right ventricle interventricular septum plus a standard implanted cardiac defibrillation with generator in left pectoral region and single implanted cardiac defibrillation lead in the right ventricle. 
were enrolled and had CCM device (with three leads) implanted. Patients were assessed at baseline, 3 and 6 months with 6MWT, MLHFQ, echocardiogram, peak $\mathrm{VO}_{2}$ and Holter monitor. In half of the patients, the device was turned on. The primary safety outcome was any hospitalization. Patients with the CCM device had a significantly decreased event-free survival (i.e., less hospitalizations). No significant difference was seen in Holter monitor recordings. The study was not powered to evaluate clinical efficacy. There was a trend of clinical improvement, but no statistical significance between control and CCM therapy patients.

\section{FIX-HF-4}

The safety and efficacy of CCM was further established in a feasibility study with a randomized, double-blind, crossover design in the FIX-HF 4 trial conducted in Europe and published in 2008 [9]. Inclusion criteria included symptomatic heart failure ( $\mathrm{New}$ York Heart Association functional class $\geq \mathrm{II}$ ), $\mathrm{LVEF} \leq 35 \%$ and peak $\mathrm{VO}_{2}$ between 10 and $20 \mathrm{ml} \mathrm{O}_{2} / \mathrm{min} / \mathrm{kg}$. Patients were required to be on OMT including diuretic, a $\beta$-blocker and an angiotensinconverting enzyme inhibitor or angiotensin-receptor blocker. Pre-existing ICD or pacemaker was allowed. Exclusion criteria included CRT device, atrial fibrillation or $\geq 8900$ PVCs on a 24-h Holter monitor. 164 patients were enrolled and received CCM implantation (with three leads). Half were randomized to have the device turned on for 3 months and then off for 3 months. The other half had the device off for 3 months and then turned on for 3 months. Before, at 3 and 6 months, the enrolled patients were assessed by $6 \mathrm{MWT}$, peak $\mathrm{VO}_{2}$ and MLHFQ. The coprimary end points were improvements peak $\mathrm{VO}_{2}$ and MLHFQ. A significant placebo effect was seen in both groups at 3 months with modest improvements in peak $\mathrm{VO}_{2}(0.40$ and $0.37 \mathrm{ml} / \mathrm{kg} / \mathrm{min})$. Patients who were switched to active treatment after 3 months demonstrated maintenance or continued clinical improvement in all parameters. In those patients for whom CCM therapy was turned off after 3 months, their clinical status decreased back toward baseline. At 6 months, patients with active CCM therapy had an increased difference in peak $\mathrm{VO}_{2}$ of $0.99 \mathrm{ml} / \mathrm{kg} / \mathrm{min}$ compared with patients whose device was turned off. Importantly, there was no significant difference in adverse events between either group. Also, there was no change in ICD firing, arrhythmia burden or PVCs as measured by Holter monitor.

\section{FIX-HF-5}

The pivotal FIX-HF-5 trial, published in 2011, evaluated the safety and clinical benefits of CCM with OMT versus OMT in a large randomized, unblinded patient population [8]. An unblinded trial design was chosen primarily as the optimal way to assure device safety over the 1-year time frame. In total, 428 patients were recruited. Inclusion criteria were similar to FIX-HF-4 trial except NYHA class limited to III or IV, EF $\leq 35 \%$ and extended peak $\mathrm{VO}_{2}$ to include $\geq 9 \mathrm{ml} / \mathrm{kg} / \mathrm{min}$. The study's primary clinical end point was $\geq 20 \%$ improvement in ventilatory anaerobic threshold (VAT). VAT is considered to be an unbiased measure of exercise tolerance not subject to the placebo effect but also has not been measured in any prior large-scale heart failure study. Before implantation, at 3 and 6 months, the enrolled patients were assessed by 6MWT, VAT, peak $\mathrm{VO}_{2}$, echocardiogram and MLHFQ. FIX-HF-5 did not meet its primary end point. However, the secondary end points were achieved with improved peak $\mathrm{VO}_{2}$ (by $0.65 \mathrm{ml} / \mathrm{kg} / \mathrm{min} ; \mathrm{p}=0.024$ ), and improved MLHFQ (by -9.7 points; $\mathrm{p}<0.0001$ ) compared with OMT. The study met the primary safety end point of noninferiority of all-cause mortality and hospitalizations compared with OMT. Interestingly, a subgroup analysis of the FIX-HF-5 trial showed that clinical benefits were more pronounced in patients with higher range LVEF $(\geq 25 \%)$ [36]. Most prominently, peak $\mathrm{VO}_{2}$ improved by $1.31 \mathrm{ml} / \mathrm{kg} / \mathrm{min}(\mathrm{p}=0.001)$ compared with OMT.

\section{Individual meta-analysis of randomized control trials}

In 2014, the meta-analysis of individual patient data from the three CCM randomized clinical trials was published [37]. Pooled analysis of 641 participants reviewed the efficacy of CMM on functional capacity, by the objective measures of peak $\mathrm{VO}_{2}$ and 6MWT and quality of life by the MLHFQ. Baseline characteristics of the participants were similar between trials. Most patients had ischemic cardiomyopathy and were NYHA class III. Overall, significant changes were seen in each parameter: peak $\mathrm{VO}_{2}$ increased by $+2.6 \%(14.5-14.7 \mathrm{ml} / \mathrm{kg} / \mathrm{min}$; $\mathrm{p}=0.006)$, 6MWT distance improved by $+10 \%(344-367 \mathrm{~m} ; \mathrm{p}=0.05)$ and MLHFQ improved by -22\% (5540; $\mathrm{p}<0.0001$ ). In subgroup analysis, patients older than 60 and patients with LVEF 25-45\% had significantly improved peak $\mathrm{VO}_{2}$. Significant improvement in 6MWT was seen in the subgroups of male gender, patients with ischemic cardiomyopathy, and LVEF $25-45 \%$. With MLHFQ, all age groups and both genders had significant 
improvement. Patients with ischemic etiology and LVEF less than $25 \%$ also had significant improvement on MLHFQ.

\section{FIX-HF-5C}

Based on the FIX-HF-5 and the individual meta-analysis observations, the FIX-HF-5 confirmatory study published in 2018 was designed to address CCM benefits in the subgroups that emerged as the high responders in the FIXHF-5 study: specifically, those with mild-to-moderate reduction in LVEF (25-45\%) [38]. Inclusion criteria were similar to previous studies: NYHA class III or IV symptoms despite OMT, EF 25-45\% and normal sinus rhythm with QRS $\leq 130$ ms. Patients with EF less than 35\% were required to have an ICD. 160 patients were enrolled in the USA and EU in a randomized, unblinded fashion. Patients were randomized to continued OMT $(\mathrm{n}=86)$ or CCM $(\mathrm{n}=74)$. Patients were assessed with peak $\mathrm{VO}_{2}$ (the primary end point), MLHFQ, NYHA functional class and 6MWT. Assessments were completed at baseline and then at 3-and 6-month postimplantation of the three lead Optimizer IV CE system. The study met its primary end point with an improvement in peak $\mathrm{VO}_{2}$ with a difference between groups of $0.84 \mathrm{ml} \mathrm{O} / \mathrm{kg} / \mathrm{min}$ (95\% Bayesian credible interval: 0.123-1.552). Statistically significant differences were also seen in quality of life by MLHFQ with -11.7 point improvement $(\mathrm{p}<0.001)$. Patients with CCM were 5.97-times more likely to improve at least one NYHA functional class compared with OMT ( $<$ 0.001). The average 6MWT improved by 43 meters $(\mathrm{p}=0.02)$. In FIX-HF-5 and FIX-HF-5C, there were 96 patients with EF greater than 35\% (49 OMT, $47 \mathrm{CCM}$ ). In comparison, 275 patients had EF less than $35 \%$ (145 OMT, 130 CCM). In patients with EF greater than 35-45\%, better CCM efficacy results were seen in each parameter with pronounced improvement in peak $\mathrm{VO}_{2}$ to $1.76 \mathrm{ml} \mathrm{O} / \mathrm{kg} / \mathrm{min}$ difference from control $(p=0.009)$. Furthermore, the composite end point of cardiovascular deaths and heart failure hospitalizations was lower in the CCM group with a great than 50\% reduction from 10.8 to $2.9 \%$ event rate. Thus, FIX-HF-5C showed that CCM improves exercise tolerance and quality of life when compared with OMT and led to fewer hospitalizations.

\section{Long-term outcomes}

While randomized clinical trials have shown improvement in heart failure morbidity end points, CCM survival benefits and long-term sustainability of clinical effects have remained not fully addressed in a prospective fashion due to short-term follow-up of prior randomized clinical trials (Table 1) $[8-10,12,16]$. Both retrospective and prospective observational studies start to address these questions.

A retrospective study of 68 patients with a CCM device and mean follow-up of 4.5 years demonstrated lower mortality rates than predicted by the Seattle Heart Failure Model (SHFM) [39]. Kaplan-Meier analysis of mortality rate of $14.2 \%$ at 5 years versus predicted $27.7 \%$ by SHFM. In single-center retrospective study, 81 patients with CCM sustained improvements in EF, quality of life (MLHFQ), symptoms, exercise tolerance through an average of 3-year follow-up. These patients had mortality rates lower than predicted by the Meta-Analysis Global Group in Chronic (MAGGIC) heart failure score. However, there was not a significant change in peak $\mathrm{VO}_{2}$ [40].

\section{CCM-REG}

The largest study to address long term outcomes is the $C C M-R^{2} G_{25-45}$. CCM-REG $25-45$ is a multicenter prospective observational registry designed to address long-term (3-year) mortality of patients who received CCM as part of routine clinical indication in Europe compared with the predicted mortality assessed by the SHFM [41]. 140 were enrolled in the overall cohort $\left(C C M-R^{2} G_{25-45}\right)$, among them: 83 patients were in the CCM-REG $25-34$ subgroup and 57 patients were in the CCM-REG $34-45$ subgroup. Patients were well treated in accordance to GDMT at time of enrollment ( $>90 \%$ received RAAS inhibitors and $\beta$ blockers). The study showed that observed survival in the overall group (CCM: 25-45\%) and in the lower LVEF group (CCM: 25-34\%) were similar to predicted survival by the SHFM (P: NS for both). Interestingly, in the CCM-REG 35-45\% group, observed survival was significantly higher than predicted survival by the SHFM ( $\mathrm{p}=0.046)$. The study also showed significant improvement in NYHA functional class and quality of life assessed by MLHFQ, as well as marked reduction in heart failure hospitalization rate in the 2 years following CCM implant across all LVEF ranges.

The study results provide further insight into the clinical benefits of CCM among patients selected in a practice setting and strengthen CCM position as an emerging therapy in heart failure therapeutic algorithm. In this study, the investigators have not only emphasized the outcome of prior randomized studies that substantiated CCM effectiveness in reducing heart failure hospitalization and improving symptoms and quality of life in patient with 


\begin{tabular}{|c|c|c|c|c|c|c|}
\hline Trial name & $\begin{array}{l}\text { Patients } \\
\text { enrolled and } \\
\text { follow-up }\end{array}$ & Inclusion & Design & End points & Results & Ref. \\
\hline Pilot study & $\begin{array}{l}49 \\
\text { (6 months) }\end{array}$ & $\begin{array}{l}\text { NYHA class III or } \\
\text { IV } \\
\text { LVEF }<35 \% \\
\text { QRS }<130 \mathrm{~ms}\end{array}$ & $\begin{array}{l}\text { Randomized, } \\
\text { double-blind, } \\
\text { pilot study }\end{array}$ & $\begin{array}{l}\text { NYHA class } \\
6 \mathrm{MWT} \\
\text { Cardiopulmonary stress test } \\
\text { (peak } \mathrm{VO}_{2} \text { ) } \\
\text { MLHFQ } \\
\text { Holter monitoring }\end{array}$ & $\begin{array}{l}6 \mathrm{MWT} \text {, peak } \mathrm{VO}_{2} \text { and anaerobic threshold } \\
\text { increased more in the treatment group than in } \\
\text { control, but were not statistically significant } \\
\text { (small sample size) } \\
\text { NYHA and MLHFQ improved similarly in the } \\
\text { two groups } \\
\text { More patients in the treatment group were } \\
\text { free of hospitalization for any cause at } \\
6 \text { months } \\
\text { No change in ectopy was observed }\end{array}$ & [35] \\
\hline FIX-HF-4 & $\begin{array}{l}164 \\
\text { (3 months) }\end{array}$ & $\begin{array}{l}\text { NYHA class II or } \\
\text { III } \\
\text { LVEF } \leq 35 \% \\
\text { QRS }<130 \mathrm{~ms}\end{array}$ & $\begin{array}{l}\text { Randomized, } \\
\text { double-blind, } \\
\text { crossover study }\end{array}$ & $\begin{array}{l}\text { Peak } \mathrm{VO}_{2} \\
\text { MLHFQ } \\
6 \mathrm{MWT} \\
\text { Safety (adverse events) }\end{array}$ & $\begin{array}{l}\text { Exercise tolerance (peak } \mathrm{VO}_{2} \text { ) and quality of } \\
\text { life (MLHFQ) were significantly better, while } \\
\text { patients were receiving active treatment with } \\
\mathrm{CCM} \text { for a 3-month period } \\
\text { No significant differences between ON and } \\
\text { OFF phases in the number or types of adverse } \\
\text { events }\end{array}$ & [9] \\
\hline FIX-HF 5 & $\begin{array}{l}428 \\
\text { ( } 6 \text { months for } \\
\text { VAT, Peak VO }{ }_{2} \text {, } \\
\text { MLWFQ) } \\
\text { ( } 12 \text { months for } \\
\text { all-cause } \\
\text { mortality and } \\
\text { hospitalization) }\end{array}$ & $\begin{array}{l}\text { NYHA class III or } \\
\text { IV } \\
\text { LVEF } \leq 35 \% \\
\text { QRS }<130 \mathrm{~ms}\end{array}$ & $\begin{array}{l}\text { Prospective, } \\
\text { randomized } \\
\text { study comparing } \\
\text { CCM plus OMT } \\
\text { to OMT alone }\end{array}$ & $\begin{array}{l}\text { Primary clinical: } \\
\text { - VAT } \\
\text { Secondary clinical: } \\
\text { - Peak } \mathrm{VO}_{2} \\
\text { - MLHFQ } \\
\text { Primary safety: } \\
\text { - Composite } 12 \text {-month } \\
\text { noninferiority of all-cause } \\
\text { mortality and } \\
\text { hospitalizations, } 12.5 \% \\
\text { allowable delta }\end{array}$ & $\begin{array}{l}\text { While VAT did not improve at } 6 \text { months, CCM } \\
\text { significantly improved peak } \mathrm{VO}_{2} \text { over OMT } \\
48 \% \text { of OMT and } 52 \% \text { of } \mathrm{CCM} \text { patients } \\
\text { experienced a safety end point, which satisfied } \\
\text { the noninferiority criterion }\end{array}$ & [8] \\
\hline FIX-HF-5C & $\begin{array}{l}160 \\
\text { (6 months) }\end{array}$ & $\begin{array}{l}\text { NYHA class III or } \\
\text { IV } \\
\text { EF } 25-45 \% \\
\text { QRS }<130 \mathrm{~ms}\end{array}$ & $\begin{array}{l}\text { Prospective, } \\
\text { randomized } \\
\text { study of } \\
\text { comparing CCM } \\
\text { plus OMT to } \\
\text { OMT alone }\end{array}$ & $\begin{array}{l}\text { Primary clinical: } \\
- \text { Peak } \mathrm{VO}_{2} \\
-6 \mathrm{MWT} \\
\text { Primary safety: } \\
\text { - Composite of } \\
\text { cardiovascular death and } \\
\text { heart failure hospitalizations }\end{array}$ & $\begin{array}{l}\text { The difference in peak } \mathrm{VO}_{2} \text { between groups } \\
\text { was } 0.84(95 \% \mathrm{Bayesian} \text { credible interval: } \\
0.123-1.552) \mathrm{ml} \mathrm{O}_{2} / \mathrm{kg} / \mathrm{min} \text {, satisfying the } \\
\text { primary end point } \\
\mathrm{MLHFQ}, \mathrm{NYHA} \text { functional class and } 6 \text {-min hall } \\
\text { walk all improved in the treatment versus } \\
\text { control group } \\
\text { The composite of cardiovascular death and } \mathrm{HF} \\
\text { hospitalizations was reduced from } 10.8 \text { to } \\
2.9 \%(\mathrm{p}=0.048)\end{array}$ & [38] \\
\hline
\end{tabular}

moderately reduced EF and narrow QRS but they also showed that those benefits were sustained after 2 years of follow-up and were present among all LVEF spectrum. Most importantly, this study establishes the long-term safety profile of CCM therapy that extends to 3 years of follow-up as compared with maximum of 1 year of follow-up in prior randomized trials [8-10].

Despite its prospective design, the study is a single-arm observational analysis that compared observed outcome against predicted model. SHFM is well-validated predictor of heart failure mortality and widely used in clinical settings. Yet the absence of an actual concurrent-matched control arm significantly weakens the validity of the study results. Objective measurement of functional capacity such as metabolic exercise testing or 6-min hallway walk could have provided more rigorous evidence of functional improvement.

\section{Expanded optimizer use}

The clinical trials to date demonstrate safety and efficacy of CCM therapy for patients with symptomatic moderateto-severe heart failure with an EF 25-45\% in normal sinus rhythm and narrow QRS ( $<130 \mathrm{~ms})$. However, CCM therapy may be beneficial patients with atrial fibrillation or patients with wide QRS ( $>130 \mathrm{~ms}$ ) based on recently published case series.

\section{Atrial fibrillation}

Timing algorithms for delivery the CCM signal rely on sequential sensing of the p-wave and depolarizations of each ventricular lead. This reliance means that patients with high burdens of atrial fibrillation were excluded from 
clinical trials. A small case series of five patients demonstrated that the algorithm could be successful adjusted to recognize paced atrial stimulation [42]. In these patients with atrial fibrillation and heart failure, they were upgraded to a CRT device with an atrial lead. The atrial sensitivity was set low resulting in compulsory atrial stimulation followed by biventricular pacing. The CCM system was implanted and recognized the atrial stimulation resulting in properly deployed CCM therapy. In this case series, patients did have improvement of clinical symptoms by NYHA class and MLHFQ. In another case series, one heart failure patient with persistent atrial fibrillation was implanted with a CCM device [43]. Because of intermittent ventricular depolarization sensing, CCM stimulation only occurred in $74 \%$ of QRS complexes. The treatment window was extended to allow for more therapeutic stimulation. The patient had improvement in his NYHA class and exercise tolerance.

\section{CRT nonresponders}

To benefit from CRT, a patient should have a left bundle-branch block (wide QRS: >130 ms), normal sinus rhythm and LVEF less than 35\% [44]. Of those with CRT, up to a third are nonresponders and do not benefit from CRT $[4,45,46]$. In a small feasibility study, CCM devices were implanted in 16 patients who did not respond to CRT and optimized medical therapy [47]. Acute CCM response was noted, but no long-term follow-up on efficacy was evaluated. No electrical interference was noted between CCM and CRT systems, but cardiac event rates were high. In a more recent open-label, treatment-only, feasibility study, 17 CRT nonresponders had CCM implanted [48]. Patients were assessed at baseline and 6 months after CCM therapy. EF trended up. Significant improvements were shown in NYHA class, MLHFQ, 6MWT and peak $\mathrm{VO}_{2}[48]$.

\section{Optimizer Smart use}

The clinical trials used to gain FDA approval of the Optimizer system used the three lead Optimizer IV CE. The current version of the device, Optimizer Smart CE, only uses two ventricular leads with an optional atrial lead. In a case series of five patients with different clinical patterns of heart failure with EF less than $35 \%$, this newest-generation Optimizer Smart CE device was used [43]. In the patient with permanent atrial fibrillation, CCM was only activated in $74 \%$ of beats during the therapy delivery interval, but the patient had improvement in both NYHA class and 6MWT. Although no improvement was seen in patients with EF less than $25 \%$ in the randomized control trial, three patients with EF of $20 \%$ and CRT nonresponders were included. One of these patients died during the study. The other two patients had striking increases in EF, NYHA and 6MWT. All four surviving patients reached NYHA functional class II from class III/IV.

\section{Future perspective}

CCM is a novel, innovative device-based therapy for heart failure recently approved by the FDA in March 2019. Based on the most recent randomized clinical trials, CCM can benefit patients with symptomatic heart failure despite optimized medical therapy with normal sinus rhythm and QRS less than $130 \mathrm{~s}$. The significance of CCM is that it provides additional device-based therapeutic benefits to patients who do not otherwise qualify for CRT (narrow QRS). CCM also expands the indication beyond the traditional LVEF cutoff of 35\% to a newer group including patients who fall in midrange LVEF group, up to $45 \%$. Importantly, these studies were conducted in era prior to extensive use of sacubitril/valsartan and ivabradine. Further prospective trials that address mortality in a prospective randomized fashion on background of current medical therapies are needed to confirm survival benefits.

With recent FDA approval, the adoption of this technology will soon be widespread (Figure 4). Infrastructure will be needed to support implementation of this novel device. Electrophysiologists will need some training in implantation of CCM devices. Device clinic staff will also need training in interrogation, evaluation and optimization of CCM. As implantation and interrogation techniques are similar to the ICD and CRT procedures, we anticipate a straightforward implementation process and infrastructure development.

Future changes can be anticipated in the refinement of this technology. A small randomized control trial of 48 patients found that the efficacy and safety of CCM was similar when the signal was delivered through a single ventricular lead rather than dual ventricular leads [49]. Thus, future models will likely consist of a single ventricular lead. In addition, a device that combines ICD and CCM in a single lead is currently under investigation. Given that many patients with an ICD could qualify for a CCM, this combination lead may become the new industry standard within the next decade. 


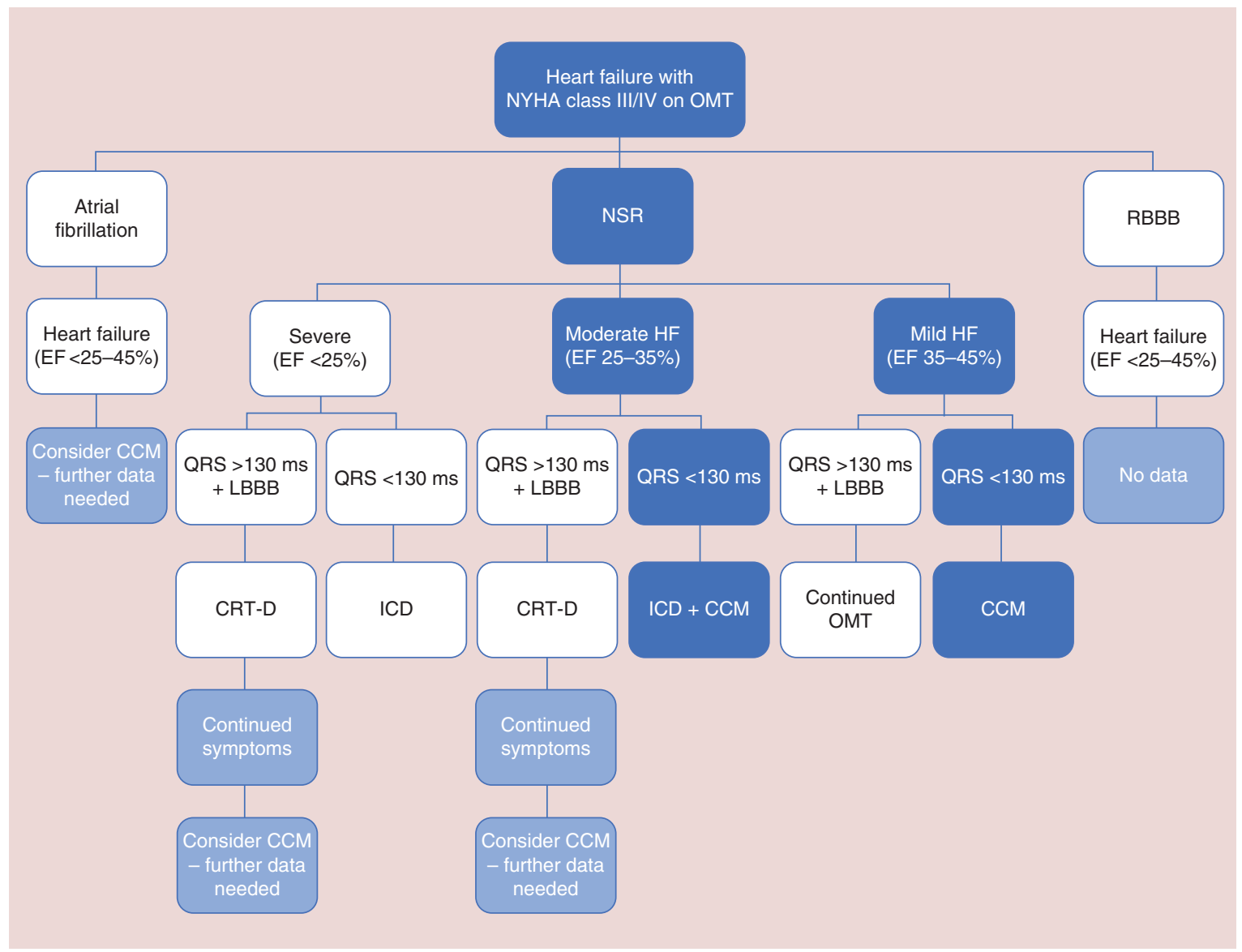

Figure 4. Intracardiac device-based therapy decision making pathway. Decision making pathway for intracardiac device-based therapy for patients with heart failure based on based on EF and electrocardiogram characteristics. Dark blue boxes indicate FDA-approved CCM indications. Light blue boxes are supported by very limited case reports and need further studies.

CCM: Cardiac contractility modulation; CRT: Cardiac resynchronization therapy; EF: Ejection fraction; HF: Heart failure; ICD: Implanted cardiac defibrillator; LBBB: Left bundle branch block; NSR: Normal sinus rhythm; NYHA: New York Heart Association; OMT: Optimal medical therapy; RBBB: Right bundle branch block.

CCM's potential benefit in atrial fibrillation is promising. In a few published cases, patients with atrial fibrillation had improvement in functional status with CCM. One patient had clinical benefit with only intermittent CCM delivery during frequent atrial fibrillation. Given that CCM therapy is only activated 8-12 h per day in patients with normal sinus rhythm, this decreased delivery percentage may not be detrimental. In contrast, CRT requires more than $90 \%$ capture for benefit, and the therapy is delivered constantly. Another alternative for patients with atrial fibrillation is to use an atrial lead with decreased atrial sensing with atrial pacing to maximize CCM delivery. Larger randomized studies should be anticipated exploring this potential benefit group.

Patients who currently qualify for CRT should be considered in future studies. Nonexcitatory CCM therapy is distinctly different than excitatory CRT. Theoretically, there is no reason both therapies cannot be of benefit to the same patient. Despite the current need for up to six implanted leads, initial studies in CRT nonresponders already show promise for CCM in this population. Whether patients who benefit from CRT can also benefit from CCM remains to be evaluated and would be more feasible with a single CCM lead system. One can postulate that a combination ICD-CCM lead could become an integral part of CRT with defibrillator (CRT-D).

Patients with reduced EF and a right bundle-branch block should also be investigated. These patients have a wide QRS but do not qualify for CRT and have not been included in any of these device trials. A few cases of patients with wider QRS despite CRT show benefit $[43,47,48]$. It seems reasonable that patients with right bundle-branch block may also benefit. 
Although clinical trials did not show benefit in patients with EF less than 25\%, published cases do show some striking results in patients with these low range EFs. These results suggest that EF may not be the definitive method to determine which patients may respond to CCM therapy. Improvements in our understanding of heart failure etiology and stratification with molecular mechanisms or markers may lead to better insights into the CCM benefit population.

Lastly, CCM has global effects on the heart. All studies and trials have focused on left ventricular failure. No studies have evaluated CCM effects on patients with right ventricular failure, which has no good treatment options, or diastolic heart failure. Assessment of CCM in these patient population should not be overlooked in future endeavors.

\section{Executive summary}

\section{Cardiac contractility modulation}

- Cardiac contractility modulation, also referred to as $\mathrm{CCM}^{\mathrm{TM}}$, device sends nonexcitatory impulses that are delivered during the absolute refractory period of the myocytes' action potential.

- CCM impulses activate intracellular signals that favorably alter gene expression and enhance calcium delivery, which in turn increase myocardial contractility and cardiac efficiency without increasing metabolic demand.

- The newest generation CCM, Optimizer Smart European Conformity (CE), consists of a rechargeable implanted pulse generator, two right ventricular septal leads and an optional atrial lead.

Randomized control trials

- For patients in normal sinus rhythm with QRS less than $130 \mathrm{~ms}$ and who have left ventricular ejection fraction between 25 and $45 \%$ who remain symptomatic despite optimal medical therapy, randomized clinical trials have demonstrated safety and efficacy of CCM by increased peak ventilatory oxygen, improved Minnesota Living with Heart Failure Questionnaire scores and fewer hospitalizations.

Long-term outcomes

- Long-term safety and benefits have been evaluated in observational studies of up to 3 years, but studies to confirm survival and clinical benefits need to be completed in a prospective, randomized fashion.

\section{Expanded Optimizer Smart CE use}

- Small case report series have shown benefit in patients with atrial fibrillation and patients that did not respond to cardiac resynchronization therapy.

Future perspective

- In the next decade, CCM will see refinement including the possible addition of cardiac defibrillation capabilities, wider-spread adoption and expansion/refinement of clinical benefit for the heart failure population.

Financial \& competing interests disclosure

R Kahwash is consulting for Medtronic and William T Abraham is consulting for Impulse Dynamics. The authors have no other relevant affiliations or financial involvement with any organization or entity with a financial interest in or financial conflict with the subject matter or materials discussed in the manuscript apart from those disclosed.

No writing assistance was utilized in the production of this manuscript.

\section{Company review disclosure}

In addition to the peer-review process, with the author's consent, the manufacturer of the product discussed in this article was given the opportunity to review the manuscript for factual accuracy. Changes were made by the author at their discretion and based on scientific or editorial merit only. The author maintained full control over the manuscript, including content, wording and conclusions.

\section{References}

Papers of special note have been highlighted as: $\bullet$ of interest; $\bullet \bullet$ of considerable interest

1. Benjamin EJ, Blaha MJ, Chiuve SE et al. Heart disease and stroke statistics - 2017 update: a report from the American Heart Association. Circulation 135(10), e146-e603 (2017).

2. Higgins SL, Hummel JD, Niazi IK et al. Cardiac resynchronization therapy for the treatment of heart failure in patients with intraventricular conduction delay and malignant ventricular tachyarrhythmias. J. Am. Coll. Cardiol. 42(8), 1454-1459 (2003).

3. Young JB, Abraham WT, Smith AL et al. Combined cardiac resynchronization and implantable cardioversion defibrillation in advanced chronic heart failure: the MIRACLE ICD trial. JAMA 289(20), 2685-2694 (2003).

4. Linde C, Ellenbogen K, McAlister FA. Cardiac resynchronization therapy (CRT): clinical trials, guidelines, and target populations. Heart Rhythm 9(Suppl. 8), S3-S13 (2012). 
5. Lund LH, Jurga J, Edner M et al. Prevalence, correlates, and prognostic significance of QRS prolongation in heart failure with reduced and preserved ejection fraction. Eur. Heart J. 34(7), 529-539 (2013).

6. Ponikowski P, Voors AA, Anker SD et al. 2016 ESC Guidelines for the diagnosis and treatment of acute and chronic heart failure: the Task Force for the diagnosis and treatment of acute and chronic heart failure of the European Society of Cardiology (ESC) developed with the special contribution of the Heart Failure Association (HFA) of the ESC. Eur. Heart J. 37(27), 2129-2200 (2016).

7. Seferovic PM, Ponikowski P, Anker SD et al. Clinical practice update on heart failure 2019: pharmacotherapy, procedures, devices and patient management. An expert consensus meeting report of the Heart Failure Association of the European Society of Cardiology. Eur. J. Heart Fail. 37(27), 2129-2200 (2019).

8. Kadish A, Nademanee K, Volosin K et al. A randomized controlled trial evaluating the safety and efficacy of cardiac contractility modulation in advanced heart failure. Am. Heart J. 161(2), 329-337 e321-e322 (2011).

-• This publication describes FIX-HF-5 randomized control clinical trial for cardiac contractility modulation (CCM) demonstrating significantly improved exercise tolerance in heart failure patients with ejection fraction $(\mathrm{EF}) \leq 35 \%$ and $\mathrm{QRS}<130 \mathrm{~ms}$.

9. Borggrefe MM, Lawo T, Butter $\mathrm{C}$ et al. Randomized, double blind study of non-excitatory, cardiac contractility modulation electrical impulses for symptomatic heart failure. Eur. Heart J. 29(8), 1019-1028 (2008).

-. This publication describes the FIX-HF-4 randomized control clinical trial for CCM demonstrating improvements in exercise tolerance and quality of life in heart failure patients with $\mathrm{EF} \leq \mathbf{3 5 \%}$ and $\mathrm{QRS}<\mathbf{1 3 0} \mathrm{ms}$.

10. Abraham WT, Kuck KH, Goldsmith RL et al. A randomized controlled trial to evaluate the safety and efficacy of cardiac contractility modulation. JACC Heart Fail. 6(10), 874-883 (2018).

11. Mann JA, Abraham WT. Cardiac contractility modulation and baroreflex activation therapy in heart failure patients. Curr. Heart Fail. Rep. 16(1), 38-46 (2019).

12. Kahwash R, Burkhoff D, Abraham WT. Cardiac contractility modulation in patients with advanced heart failure. Expert Rev. Cardiovasc. Ther. 11(5), 635-645 (2013).

13. Lyon AR, Samara MA, Feldman DS. Cardiac contractility modulation therapy in advanced systolic heart failure. Nat. Rev. Cardiol.10(10), 584-598 (2013).

- This publication is an excellent review of CCM basic and translational science studies.

14. Wood EH, Heppner RL, Weidmann S. Inotropic effects of electric currents. I. Positive and negative effects of constant electric currents or current pulses applied during cardiac action potentials. II. Hypotheses: calcium movements, excitation-contraction coupling and inotropic effects. Circ. Res. 24(3), 409-445 (1969).

15. Burkhoff D, Shemer I, Felzen B et al. Electric currents applied during the refractory period can modulate cardiac contractility in vitro and in vivo. Heart Fail. Rev. 6(1), 27-34 (2001).

16. Morita H, Suzuki G, Haddad W et al. Cardiac contractility modulation with nonexcitatory electric signals improves left ventricular function in dogs with chronic heart failure. J. Card. Fail. 9(1), 69-75 (2003).

17. Imai M, Rastogi S, Gupta RC et al. Therapy with cardiac contractility modulation electrical signals improves left ventricular function and remodeling in dogs with chronic heart failure. J. Am. Coll. Cardiol. 49(21), 2120-2128 (2007).

18. Winter J, Brack KE, Ng GA. The acute inotropic effects of cardiac contractility modulation (CCM) are associated with action potential duration shortening and mediated by beta1-adrenoceptor signalling. J. Mol. Cell. Cardiol. 51(2), 252-262 (2011).

19. Kawano H, Okada R, Yano K. Histological study on the distribution of autonomic nerves in the human heart. Heart Vessels 18(1), 32-39 (2003).

20. Brunckhorst CB, Shemer I, Mika Y, Ben-Haim SA, Burkhoff D. Cardiac contractility modulation by non-excitatory currents: studies in isolated cardiac muscle. Eur. J. Heart Fail. 8(1), 7-15 (2006).

21. Mohri S, Shimizu J, Mika Y et al. Electric currents applied during refractory period enhance contractility and systolic calcium in the ferret heart. Am. J. Physiol. Heart Circ. Physiol. 284(4), H1119-H1123 (2003).

22. Gupta RC, Mishra S, Wang M et al. Cardiac contractility modulation electrical signals normalize activity, expression, and phosphorylation of the $\mathrm{Na}^{+}-\mathrm{Ca}^{2+}$ exchanger in heart failure. J. Card. Fail. 15(1), 48-56 (2009).

23. Butter C, Rastogi S, Minden HH, Meyhofer J, Burkhoff D, Sabbah HN. Cardiac contractility modulation electrical signals improve myocardial gene expression in patients with heart failure. J. Am. Coll. Cardiol. 51(18), 1784-1789 (2008).

24. Gupta RC, Mishra S, Rastogi S et al. $\mathrm{Ca}(2+)$-binding proteins in dogs with heart failure: effects of cardiac contractility modulation electrical signals. Clin. Transl. Sci. 2(3), 211-215 (2009).

25. Goliasch G, Khorsand A, Schutz M et al. The effect of device-based cardiac contractility modulation therapy on myocardial efficiency and oxidative metabolism in patients with heart failure. Eur. J. Nucl. Med. Mol. Imaging 39(3), 408-415 (2012).

26. Butter C, Wellnhofer E, Schlegl M, Winbeck G, Fleck E, Sabbah HN. Enhanced inotropic state of the failing left ventricle by cardiac contractility modulation electrical signals is not associated with increased myocardial oxygen consumption. J. Card. Fail. 13(2), 137-142 (2007).

27. Packer M, Carver JR, Rodeheffer RJ et al. Effect of oral milrinone on mortality in severe chronic heart failure. The PROMISE Study Research Group. N. Engl. J. Med. 325(21), 1468-1475 (1991). 
28. Stix G, Borggrefe M, Wolpert $\mathrm{C}$ et al. Chronic electrical stimulation during the absolute refractory period of the myocardium improves severe heart failure. Eur. Heart J. 25(8), 650-655 (2004).

29. Lawo T, Borggrefe M, Butter C et al. Electrical signals applied during the absolute refractory period: an investigational treatment for advanced heart failure in patients with normal QRS duration. J. Am. Coll. Cardiol. 46(12), 2229-2236 (2005).

30. Burkhoff D, Ben-Haim SA. Nonexcitatory electrical signals for enhancing ventricular contractility: rationale and initial investigations of an experimental treatment for heart failure. Am. J. Physiol. Heart Circ. Physiol. 288(6), H2550-H2556 (2005).

31. Borggrefe M, Mann DL. Cardiac Contractility Modulation in 2018. Circulation 138(24), 2738-2740 (2018).

32. Abraham WT, Burkhoff D, Nademanee $\mathrm{K}$ et al. A randomized controlled trial to evaluate the safety and efficacy of cardiac contractility modulation in patients with systolic heart failure: rationale, design, and baseline patient characteristics. Am. Heart J. 156(4), 641-648.e641 (2008).

33. Pappone C, Rosanio S, Burkhoff D et al. Cardiac contractility modulation by electric currents applied during the refractory period in patients with heart failure secondary to ischemic or idiopathic dilated cardiomyopathy. Am. J. Cardiol. 90(12), 1307-1313 (2002).

34. Pappone C, Augello G, Rosanio S et al. First human chronic experience with cardiac contractility modulation by nonexcitatory electrical currents for treating systolic heart failure: mid-term safety and efficacy results from a multicenter study. J. Cardiovasc. Electrophysiol. 15(4), 418-427 (2004).

35. Neelagaru SB, Sanchez JE, Lau SK et al. Nonexcitatory, cardiac contractility modulation electrical impulses: feasibility study for advanced heart failure in patients with normal QRS duration. Heart Rhythm. 3(10), 1140-1147 (2006).

-. This publication describes the pilot study for CCM, a randomized control trial demonstrating feasibility with trends toward efficiacy in heart failure patients with $\mathrm{EF}<35 \%$ and $\mathrm{QRS}<130 \mathrm{~ms}$.

36. Abraham WT, Nademanee K, Volosin K et al. Subgroup analysis of a randomized controlled trial evaluating the safety and efficacy of cardiac contractility modulation in advanced heart failure. J. Card. Fail. 17(9), 710-717 (2011).

37. Giallauria F, Vigorito C, Piepoli MF, Stewart Coats AJ. Effects of cardiac contractility modulation by non-excitatory electrical stimulation on exercise capacity and quality of life: an individual patient's data meta-analysis of randomized controlled trials. Int. J. Cardiol. 175(2), 352-357 (2014).

- This publication is an individual meta-analysis of all patients with CCM therapy in randomized control trials including the pilot study, FIX-HF 4 and FIX-HF 5.

38. Abraham WT, Lindenfeld J, Reddy VY et al. A randomized controlled trial to evaluate the safety and efficacy of cardiac contractility modulation in patients with moderately reduced left ventricular ejection fraction and a narrow QRS duration: study rationale and design. J. Card. Fail. 21(1), 16-23 (2015).

-. This publication describes the results of the FIX-HF-5C clinical trial that demonstrates CCM therapy efficacy in patients with EF $25-45 \%$ and QRS $<130 \mathrm{~ms}$.

39. Kloppe A, Lawo T, Mijic D, Schiedat F, Muegge A, Lemke B. Long-term survival with cardiac contractility modulation in patients with NYHA II or III symptoms and normal QRS duration. Int. J. Cardiol. 209, 291-295 (2016).

40. Kuschyk J, Roeger S, Schneider R et al. Efficacy and survival in patients with cardiac contractility modulation: long-term single center experience in 81 patients. Int. J. Cardiol. 183, 76-81 (2015).

41. Anker SD, Borggrefe M, Neuser $\mathrm{H}$ et al. Cardiac contractility modulation improves long-term survival and hospitalizations in heart failure with reduced ejection fraction. Eur. J. Heart Fail. 21(9), 1103-1113 (2019).

42. Roger S, Schneider R, Rudic B et al. Cardiac contractility modulation: first experience in heart failure patients with reduced ejection fraction and permanent atrial fibrillation. Europace 16(8), 1205-1209 (2014).

43. Tint D, Florea R, Micu S. New generation cardiac contractility modulation device-filling the gap in heart failure treatment. J. Clin. Med. 8(5) 588 (2019).

44. Cleland JG, Daubert JC, Erdmann E et al. The effect of cardiac resynchronization on morbidity and mortality in heart failure. $N$. Engl. J. Med. 352(15), 1539-1549 (2005).

45. Hawkins NM, Petrie MC, MacDonald MR, Hogg KJ, McMurray JJ. Selecting patients for cardiac resynchronization therapy: electrical or mechanical dyssynchrony? Eur. Heart J. 27(11), 1270-1281 (2006).

46. Birnie DH, Tang AS. The problem of non-response to cardiac resynchronization therapy. Curr. Opin. Cardiol. 21(1), 20-26 (2006).

47. Nagele H, Behrens S, Eisermann C. Cardiac contractility modulation in non-responders to cardiac resynchronization therapy. Europace 10(12), 1375-1380 (2008).

48. Kuschyk J, Nagele H, Heinz-Kuck K et al. Cardiac contractility modulation treatment in patients with symptomatic heart failure despite optimal medical therapy and cardiac resynchronization therapy (CRT). Int. J. Cardiol. 277, 173-177 (2019).

49. Roger S, Said S, Kloppe A et al. Cardiac contractility modulation in heart failure patients: randomized comparison of signal delivery through one vs. two ventricular leads. J. Cardiol. 69(1), 326-332 (2017). 
